\title{
Erratum to: Multi-objective Topic Modeling for Exploratory Search in Tech News
}

Anastasia Ianina, Lev Golitsyn, and Konstantin Vorontsov

\author{
Erratum to: \\ Chapter "Multi-objective Topic Modeling for Exploratory \\ Search in Tech News" in: A. Filchenkov et al. (Eds.): \\ Artificial Intelligence and Natural Language, CCIS 789, \\ https://doi.org/10.1007/978-3-319-71746-3_16
}

The original version of this book has been revised. The Acknowledgements section on page 192 of paper https://doi.org/10.1007/978-3-319-71746-3_16 has been modified.

An erratum to this book can be found at https://doi.org/10.1007/978-3-319-71746-3_16

The updated online version of this chapter can be found at https://doi.org/10.1007/978-3-319-71746-3_16

(C) Springer International Publishing AG 2018

A. Filchenkov et al. (Eds.): AINL 2017, CCIS 789, p. E1, 2018.

https://doi.org/10.1007/978-3-319-71746-3_24 